\title{
Super-heróis em educação nutricional: o lúdico na promoção da saúde e prevenção da anemia em pré-escolares
}

\author{
Superheroes in nutrition education: the ludic in health promotion and
} prevention of anemia in preschool children

\author{
Samara de Almeida Mesquita Rosa* \\ Márcia Rubia Duarte Buchweitz** \\ Dionísia Nagahama*** \\ Derlange Belizário Diniz****
}

\begin{abstract}
Resumo
O estudo avaliou o impacto de uma intervenção em educação nutricional sobre o conhecimento e atitudes de pré-escolares visando o estímulo de hábitos alimentares saudáveis e prevenção da anemia. Houve avaliação comparativa pré e pós-atividade educativa, com crianças entre 2 e 6 anos de idade, durante 12 meses. A metodologia de ensino foi baseada na aprendizagem por modelação, com personagens representando a anemia e os super-heróis ferro, vitamina C e a saúde. Foram utilizados reforçadores positivos visando sedimentação do aprendizado. Para mensurar a incorporação dos conhecimentos e aceitação das preparações com alimentos fontes de ferro e vitamina C, utilizou-se cartilha educativa e análise da satisfação quanto aos cardápios propostos. A maioria $(62,74 \%)$ respondeu adequadamente sobre como combater a anemia, citando abordagens educativas utilizadas durante a intervenção. A baixa aceitação inicial das refeições caiu significativamente $(p<0,05)$. O desenho em estilo livre sobre os alimentos incentivados quintuplicou após a intervenção $(14,7 \%$ para $76 \%)$. Concluiu-se que a utilização de uma metodologia lúdica associando super-heróis e reforçadores positivos apresenta impacto considerável na assimilação do conhecimento sobre alimentação saudável e seu efeito na saúde em crianças pré-escolares. Concluiu-se que a utilização de uma metodologia lúdica associando super-heróis e reforçadores positivos apresenta impacto considerável na assimilação do conhecimento sobre alimentação saudável e seu efeito na saúde em crianças pré-escolares.
\end{abstract}

Palavras-chave: Educação Nutricional. Pré-escolar. Comportamento Alimentar. Super-heróis.

\begin{abstract}
This study measured the impact of the nutritional education's intervention on the preschool children's knowledge and actions, aiming both for a promotion of a healthy eating habits and for a prevention of iron deficiency anemia. There was a comparative evaluation before and after educational activities during 12 months in a kindergarten in the city of Fortaleza, CE. The education's methodology was grounded on learning through modeling, with characters representing anemia and superheroes representing iron, vitamin $C$ and health. Positive reinforcements aiming learning sedimentation were used. To measure the rate of the knowledge absorption and the acceptance of the recipes it was used tools such as an educational booklet along with sensory analysis of the new menu's acceptance. The majority $(62,74 \%)$ answered clearly about how to prevent anemia, referring about the approaches used during the intervention. The meals' rejection significantly dropped $(p<0,05)$. The freestyle drawing about the encouraged food quintupled after the intervention $(14,7 \%$ to $76 \%)$.It was concluded that the use of characters from children's imagination and the positive reinforcements improved their learning, encouraging healthier eating habits.
\end{abstract}

Keywords: Nutrition Education. Preschool. Feeding behavior. Superheroes.

DOI: $10.15343 / 0104-7809.20153904495503$

\# A pesquisa teve apoio financeiro do Conselho Nacional de Desenvolvimento Científico e Tecnológico - CNPq (proc. nº 483794/2010-4). O estudo resultou da dissertação apresentada ao Curso de Mestrado Acadêmico em Nutrição e Saúde do Centro de Ciências da Saúde da Universidade Estadual do Ceará com título "O Lúdico como estratégia de educação nutricional: promovendo a alimentação saudável e prevenindo a anemia em crianças da pré-escola" com autoria de Samara de Almeida Mesquita Rosa e orientação de Derlange Belizário Diniz.

* Centro Universitário Estácio do Ceará. Fortaleza - CE, Brasil. E-mail: marameida@yahoo.com.br

** Universidade Federal de Pelotas, Pelotas - RS, Brasil. E-mail: marciabuchweitz@gmail.com

*** Instituto Nacional de Pesquisas da Amazônia. Manaus - AM, Brasil. E-mail: naga@inpa.gov.br

**** Universidade Estadual do Ceará. Fortaleza - CE, Brasil. E-mail: derlange.diniz@uece.br

Os autores declaram não haver conflitos de interesse. 


\section{INTRODUÇÃO}

A educação nutricional apresenta resultados extremamente positivos quando desenvolvida na infância, pois os primeiros anos de vida são responsáveis pela construção de diversos hábitos, aprendidos a partir da experiência e da observação ${ }^{1,2}$. Há evidências que os hábitos alimentares adquiridos na infância interferem a promoção de uma melhor qualidade de vida na adolescência e vida adulta ${ }^{3}$.

Os esforços para melhorar a nutrição e sua influência na construção dos hábitos infantis podem ser particularmente eficazes ${ }^{4}$. A importância de intervenções nutricionais cada vez mais precoces é reforçada pelo atual processo de transição epidemiológica em que se encontra a população brasileira, com predominância de doenças crônicas não transmissíveis, cuja morbimortalidade está associada à alimentação e estilo de vida. Concomitante a esse quadro, persiste ainda uma realidade de carências nutricionais ${ }^{5}$.

Deficiências nutricionais como a carência de ferro, gera grande preocupação em saúde pública, pois as crianças em idade pré-escolar constituem um grupo altamente vulnerável, sendo expostas aos prejuízos acarretados em seu desenvolvimento ${ }^{1,6}$. A anemia interfere negativamente sobre o crescimento ponderal e estatural, na aprendizagem e desenvolvimento psicomotor, podendo persistir mesmo após uma suplementação ferropriva ${ }^{7}$. A mudança de comportamento visando evitar tais consequências deve utilizar estratégias que envolva diversos atores, como a família, a equipe de educadores e colaboradores da instituição de ensino ${ }^{8}$.

A escola tem se mostrado como o ambiente mais favorável para o desenvolvimento de ações em educação nutricional, pois envolve o escolar, o núcleo familiar e a comunidade, influenciando positivamente o comportamento alimentar.9, 10 .

Segundo as diretrizes da Política Nacional de Alimentação e Nutrição (PNAN), o espaço escolar deve ser incentivado como ambiente de prática para educação nutricional, inserindo a alimentação e nutrição no conteúdo programático

em diferentes níveis de ensino e respeitando hábitos regionais ${ }^{11}$. O Programa Nacional de Alimentação Escolar (PNAE) do governo brasileiro estabelece, por sua vez, valores de suprimento parcial das necessidades nutricionais dos escolares, dependendo do tipo e número de refeições realizadas na escola ${ }^{12}$.

O desenvolvimento de um trabalho educativo com abordagem específica para o público infantil proporciona a ampliação do conhecimento das crianças e seus familiares em torno dos cuidados com a alimentação e nutrição, favorecendo escolhas alimentares mais saudáveis e valorizando a utilização da alimentação escolar ${ }^{4}$.

As atividades de educação devem levar em consideração o perfil nutricional dos escolares, além dos aspectos sociais, culturais e econômicos, promovendo de forma eficaz a formação de hábitos alimentares adequados e prevenção de doenças relacionadas à má alimentação ${ }^{11}$.

As crianças descobrem o seu ambiente através dos sentidos, utilizando suas experiências na formação da memória. No contexto da educação nutricional, as crianças em idade pré-escolar têm limitações para compreender o papel dos nutrientes e sua relação com os alimentos, ou sobre o conceito de saúde. No entanto, estratégias que transformem esses conceitos em figuras do imaginário infantil podem auxiliar o processo de aprendizagem ${ }^{13,14}$. Destaca-se a mudança por modelagem do comportamento, em que a criança reproduz determinada ação realizada por um personagem infantil, amigo, familiar ou mesmo o professor ${ }^{15,16,17}$.

O processo de aprendizagem lúdica também pode favorecer o desenvolvimento da linguagem, do pensamento, da socialização, da iniciativa e da autoestima, preparando o indivíduo para ser um cidadão apto a transpor dificuldades e ser mais seguro em fazer escolhas. O brincar e o jogar são atos imprescindíveis à saúde física, emocional e intelectual do ser humano ${ }^{4}$. O presente estudo baseou-se na utilização da figura de super-heróis como estratégia de educação nutricional com o objetivo de promover a alimentação saudável e prevenir a anemia ferropriva. 


\section{MÉTODO}

O estudo foi realizado em uma pré-escola comunitária de Fortaleza, Ceará após ser aprovado pelo Comitê de Ética em Pesquisa da Universidade Estadual do Ceará sob parecer consubstanciado n. 11044527-9. A escola atendia crianças com idade entre 2 e 6 anos, provenientes de famílias de baixo poder aquisitivo.

Participaram da pesquisa crianças que faziam pelo menos duas refeições na escola, sendo uma delas almoço ou jantar. Foram excluídas da pesquisa as crianças que tiveram frequência escolar inferior a $60 \%$ no período do estudo.

O cardápio da escola foi ajustado para atender ao preconizado pelo PNAE, com a introdução de novas preparações e alimentos, de forma a adequar a ingestão de calorias totais e nutrientes, especificamente quanto a ferro e vitamina $\mathrm{C}^{12}$.

Procurou-se incluir alimentos de baixo custo e de fácil aquisição como as frutas sazonais, os alimentos disponíveis em mercados locais ou fornecidos pela prefeitura municipal. Os manipuladores de alimentos da escola foram treinados para executar o novo cardápio.

De um modo geral foi acrescentada aos lanches a carne moída, ovos, cuscuz e massa de cereal enriquecido com ferro. Ao lanche da tarde foram incluídas vitaminas de frutas e bolo de rapadura. No almoço foram introduzidos fígado, couve-manteiga, feijão, ovos, acompanhados com rapadura, frutas da época, sucos de acerola, laranja, limão, goiaba e caju. No jantar foi adicionado fígado na sopa de feijão.O estudo ocorreu em três fases:

\section{$1^{\text {a }}$ fase - Sensorial.}

Foi realizada avaliação sensorial da aceitação dos cardápios. O monitoramento consistiu na utilização de um cartão individual, com escala hedônica em três faixas de aceitação: alta (100\% a $75 \%)$, baixa (25\%) e rejeição $(0 \%)$. $\mathrm{O}$ instrumento foi preenchido pela professora responsável, observando a criança durante as refeições. O monitoramento ocorreu em três momentos: nos cinco primeiros dias, durante a intervenção e na fase de manutenção.

\section{$2^{\mathrm{a}}$ fase - Intervenção.}

Essa fase teve a duração de sete semanas e foram aplicadas as atividades lúdicas (Figura 1), segundo os fundamentos da aprendizagem por modelação ${ }^{15,16}$. Foram criados super-heróis, denominados Nutri Heróis, representados pela saúde (Bela Saúde), o ferro (Super Ferro) e a vitamina C, que combatiam o vilão (Pavoroso Anêmico). O vídeo e música (Nutri Heróis: os protetores da saúde) estão disponíveis no endereço eletrônico http:// www.youtube.com/watch?v=yTNG2GNJov0.

A modelagem consistiu na utilização de diversos materiais e dinâmicas educativas, como réplicas de alimentos fontes de ferro e vitamina $C$, peças de teatro, brincadeiras, álbum de figurinhas e cartilhas de atividades (Tabela 1).

Pesquisadores fantasiados de Nutri Heróis e de Pavoroso Anêmico realizaram atividades em sala de aula empregando os diversos materiais produzidos. Alguns desses materiais foram aplicados como reforços positivos para fortalecer a aprendizagem.

As réplicas de alimentos representavam os alimentos introduzidos no cardápio da escola: fígado, folha verde escura, rapadura, acerola, caju, goiaba, laranja e limão, além da carne, do feijão e do ovo, cujo consumo foi estimulado.

A cartilha de atividades, com diversos tipos de exercícios incluía pintura de personagens e alimentos, liga-pontos, labirinto, completa-palavras e desenho livre, associados à figura dos nutri heróis no combate a anemia.

Três vezes por semana eram realizadas atividades abordando o tema, sendo uma delas envolvendo a participação dos personagens em dinâmicas teatrais. Nas outras duas vezes, os professores estimulavam as crianças por meio de exercícios com a cartilha de atividades, assistindo o desenho animado e cantando a música dos Nutri Heróis.

\section{$3^{\mathrm{a}}$ fase - Manutenção.}

Teve a duração de três meses. Nesse período as crianças receberam como reforçador positivo um álbum de figurinhas. Semanalmente eram entregues figuras que reforçavam os conhecimentos sobre higiene pessoal, alimentos fontes de ferro, vitamina C e os Nutri Heróis. 
Tabela 1. Descrição das atividades realizadas no período da intervenção com pré-escolares de uma escola comunitária de educação infantil. Fortaleza, CE. 2013

\begin{tabular}{|c|c|c|c|}
\hline ATIVIDADE & OBJETIVO & METODOLOGIA & PERÍODO \\
\hline $\begin{array}{l}\text { Teatro de } \\
\text { Personagens }\end{array}$ & $\begin{array}{l}\text { Apresentar história com os } \\
\text { personagens, seus alimentos fontes } \\
\text { e as complicações da anemia }\end{array}$ & $\begin{array}{l}\text { Teatro com os personagens e alimentos } \\
\text { fontes de ferro e vitamina C }\end{array}$ & $1^{\text {a }}$ Semana \\
\hline Bola ao Cesto & $\begin{array}{l}\text { Associar os personagens/nutrientes } \\
\text { com seus alimentos fontes }\end{array}$ & $\begin{array}{l}\text { Brincadeira com bolas representando os } \\
\text { alimentos e cestos personalizados com } \\
\text { os Nutri Heróis }\end{array}$ & $2^{\text {a Semana }}$ \\
\hline $\begin{array}{l}\text { Aprendendo a } \\
\text { lavar as mãos }\end{array}$ & $\begin{array}{l}\text { Ensinar noções de higiene como } \\
\text { lavar as mãos }\end{array}$ & Teatro interativo e Pintura de mãos & $3^{\text {a Semana }}$ \\
\hline Jogo da Memória & $\begin{array}{l}\text { Memorizar os alimentos fontes de } \\
\text { ferro e vitamina } C\end{array}$ & $\begin{array}{l}\text { Jogo da memória utilizando cartas } \\
\text { gigantes }\end{array}$ & $4^{\mathrm{a}}$ Semana \\
\hline Caça ao tesouro & $\begin{array}{l}\text { Estimular o interesse pelos alimentos } \\
\text { que combatem a anemia }\end{array}$ & $\begin{array}{l}\text { Encontrar os alimentos escondidos pelo } \\
\text { "Pavoroso Anêmico" e entregavam aos } \\
\text { respectivos Nutri Heróis }\end{array}$ & $5^{\text {a Semana }}$ \\
\hline Lanche Saudável & $\begin{array}{l}\text { Conhecer os alimentos } \\
\text { fontes de ferro e vitamina } \mathrm{C} \\
\text { experimentando-os in natura }\end{array}$ & $\begin{array}{l}\text { Apresentação de alguns alimentos fontes } \\
\text { de ferro e vitamina C através da visão, } \\
\text { tato, olfato e paladar }\end{array}$ & $6^{\text {a Semana }}$ \\
\hline Estátua & $\begin{array}{l}\text { Reforçar o aprendizado utilizando } \\
\text { a música }\end{array}$ & $\begin{array}{l}\text { Interação entre os personagens e as } \\
\text { crianças utilizando a música tema }\end{array}$ & $7^{\mathrm{a}}$ Semana \\
\hline Cartilha & $\begin{array}{l}\text { Sedimentar o conhecimento } \\
\text { passado pelos personagens }\end{array}$ & $\begin{array}{l}\text { Desenho livre, pintura, ligando pontos, } \\
\text { labirinto, e outras atividades envolvendo } \\
\text { os personagens e os alimentos }\end{array}$ & $\begin{array}{l}\text { Duas vezes } \\
\text { na semana }\end{array}$ \\
\hline Desenho Animado & $\begin{array}{l}\text { Apresentação dos Nutri Heróis } \\
\text { combatendo as doenças }\end{array}$ & $\begin{array}{l}\text { Apresentação do desenho animado em } \\
\text { salas de aula pelos professores }\end{array}$ & $\begin{array}{c}\text { Fase de } \\
\text { manutenção }\end{array}$ \\
\hline $\begin{array}{l}\text { Música dos Nutri } \\
\text { Heróis - }\end{array}$ & $\begin{array}{l}\text { Ensinar sobre alimentos fontes de } \\
\text { ferro e vitamina } C\end{array}$ & Cantar a música com as crianças & $\begin{array}{l}\text { Durante as } \\
\text { atividades }\end{array}$ \\
\hline
\end{tabular}

Paralelamente, foram realizados oito encontros com os pais/responsáveis, abordando temas sobre alimentação saudável, higiene pessoal, ambiental e dos alimentos, causas e consequências da anemia, e oficinas de preparação de receitas com alimentos fontes de ferro e vitamina C. Como reforçadores de conteúdo foram distribuídos imãs e receitas culinárias.

\section{Análise da Incorporação da Ação Educativa}

Antes das fases de Intervenção e Manutenção os professores aplicaram uma atividade de desenho livre, com o tema: como ajudar os Nutri Heróis na luta contra o Pavoroso Anêmico. Essa mesma atividade foi aplicada ao final do estudo. Os professores identificaram o significado dos desenhos conforme o relato das crianças. A comparação entre os desenhos dos dois momentos teve como objetivo avaliar a incorporação do conhecimento transmitido.

Ao final da fase de manutenção as perguntas foram feitas para cada criança, em sala isolada, com registro escrito fidedigno à resposta, seguindo proposta de Jorge $(2006)^{18} \mathrm{e}$ Plum $(1997)^{19}$, com adaptações. Os relatos foram categorizados em temas predominantes e posteriormente analisados.

\section{Análise estatística}

Para caracterização da amostra as variáveis foram apresentadas como médias, desvios-padrão e proporções. Para testar a associação entre variáveis categorizadas foi utilizado o teste qui quadrado. Em todos os testes estatísticos, o nível de significância foi igual ou inferior a 5\%. O software utilizado foi o SPSS versão 13.0. 


\section{RESULTADOS}

O estudo foi realizado em uma pré-escola do município de Fortaleza, Ceará, de acordo com os seguintes critérios de elegibilidade: ser comunitária, oferecer refeições aos alunos, atender crianças de 2 a 6 anos e que consentisse receber a intervenção educativa, comprometendo-se a adquirir e incluir no cardápio os alimentos fon- tes de ferro propostos. O período estabelecido para realização do estudo foi de 12 meses, entre 2011 e 2012.

Participaram do estudo 78 crianças, com idade média de $55 \pm 14$ meses, sem diferença significativa entre os sexos. Em relação à alimentação oferecida, na Tabela 2 verificou-se que a aceitação melhorou significativamente $(p \leq 0,05)$.

Tabela 2. Aceitação do cardápio antes e após a estratégia educativa em pré-escolares de uma escola de educação infantil. Fortaleza, CE. 2013

\begin{tabular}{lccccc}
\hline Refeições & $\begin{array}{c}\text { Aceitação } \\
\%\end{array}$ & $\begin{array}{c}\text { Antes } \\
\%\end{array}$ & $\begin{array}{c}\text { Durante } \\
\%\end{array}$ & $\begin{array}{c}\text { Após } \\
\%\end{array}$ & p $^{*}$ \\
\hline Lanche da Manhã & Boa & 79,9 & 83,2 & 68,6 & $<0,001$ \\
& Baixa & 7,8 & 6,6 & 25,7 & \\
& Rejeição & 12,3 & 10,2 & 5,7 & \\
\hline Almoço & Boa & 82,8 & 89,9 & 87,14 & 0,489 \\
& Baixa & 13,3 & 7,9 & 8,6 & \\
& Rejeição & 3,9 & 2,2 & 4,3 & 0,003 \\
Lanche da Tarde & Boa & 89,1 & 95,7 & 96,7 & \\
& Baixa & 7,8 & 3,2 & 2,0 & $<0,001$ \\
\hline Jantar & Rejeição & 3,0 & 1,1 & 1,3 & \\
& Boa & 64,8 & 81,8 & 76,4 & \\
& Baixa & 9,9 & 4,3 & 3,8 & 19,8 \\
\hline
\end{tabular}

* Teste $x^{2}$

Para todas as refeições houve um aumento no percentual de aceitação comparando-se os momentos antes e durante a intervenção. Esse resultado também se manteve quando foram comparados os momentos antes e após intervenção, exceto para o lanche da manhã. No entanto, para esse último, se observa que o percentual de rejeição foi menor após a ação educativa.

Os desenhos livres feitos pelas crianças antes e após a intervenção (Tabela 3), em que era solicitado demonstrar "como ajudar o Super Ferro e a Vitamina C a combater o vilão Pavoroso Anêmico", revelaram que houve um aumento significativo do número de desenhos associados aos personagens e alimentos fontes de ferro e vitamina $C$ após a intervenção, passando de $14,7 \%$ para $76 \%(p=0,000)$. Antes da intervenção, 85,3\% desenharam apenas um ou nenhum alimento sem relação com o combate à anemia. Após a intervenção, houve prevalência significativa de desenhos que representavam os personagens ou alimentos abordados pelos Nutri Heróis (56\%, $p=0,006)$.

Quando indagadas sobre "Como combater a anemia", observou-se uma associação positiva das respostas das crianças para um comportamento indicativo de saúde e negativo para a doença. Souberam responder claramente à pergunta $62,74 \%$ das crianças, onde $51 \%$ referiram termos como "ficar mais forte" ou "comer 
muito" $(15,7 \%)$, alimentos fontes de ferro e vitamina $\mathrm{C}(9,8 \%)$, os nomes dos personagens (3,9\%) ou a música dos Nutri Heróis $(21,6 \%)$.

O restante respondeu com expressões literais como "batendo na anemia" ou "destruindo a anemia", relacionadas diretamente ao termo "combater" utilizado na pergunta inicial (Tabela 4).

Tabela 3. Interpretação dos desenhos dos pré-escolares antes e após ação educativa em escola de educação infantil. Fortaleza, CE. 2013.

\begin{tabular}{|c|c|c|c|c|c|}
\hline \multirow{2}{*}{ Descrição } & \multicolumn{2}{|c|}{ Antes } & \multicolumn{2}{|c|}{ Após } & \multirow{2}{*}{$\mathbf{p}^{*}$} \\
\hline & $\mathbf{N}$ & $\%$ & $\mathbf{N}$ & $\%$ & \\
\hline Um ou nenhum alimento & 64 & 85,3 & 18 & 24,0 & 0,000 \\
\hline Mais de um alimento & 11 & 14,7 & 57 & 76,0 & \\
\hline Total & 75 & 100 & 75 & 100 & \\
\hline Em branco ou desenho não identificado & 18 & 24,0 & 8 & 10,7 & 0,006 \\
\hline Paisagem, sentimentos & 12 & 16,0 & 10 & 13,3 & \\
\hline Alimentos diversos & 30 & 40,0 & 15 & 20,0 & \\
\hline Personagens ou alimentos da intervenção & 15 & 20,0 & 42 & 56,0 & \\
\hline Total & 75 & 100 & 75 & 100 & \\
\hline
\end{tabular}

*Teste $x^{2}$

Tabela 4. Relação entre a idade e a resposta sobre como combater a anemia em pré-escolares de uma escola de educação infantil. Fortaleza, CE. 2013.

\begin{tabular}{|c|c|c|c|c|c|c|c|c|c|c|c|c|c|c|}
\hline \multirow[t]{2}{*}{$\begin{array}{l}\text { Idade em } \\
\text { meses }\end{array}$} & \multicolumn{2}{|c|}{ Total } & \multicolumn{2}{|c|}{$\begin{array}{l}\text { Não soube } \\
\text { responder }\end{array}$} & \multicolumn{2}{|c|}{$\begin{array}{c}\text { Alimentos } \\
\text { fontes de } \\
\text { ferro e vit C }\end{array}$} & \multicolumn{2}{|c|}{$\begin{array}{l}\text { Música dos } \\
\text { nutri heróis }\end{array}$} & \multicolumn{2}{|c|}{$\begin{array}{l}\text { Nutri } \\
\text { Heróis }\end{array}$} & \multicolumn{2}{|c|}{$\begin{array}{l}\text { Ficar forte/ } \\
\text { comer } \\
\text { muito }\end{array}$} & \multicolumn{2}{|c|}{$\begin{array}{c}\text { Destruir a } \\
\text { anemia }\end{array}$} \\
\hline & $\mathrm{n}$ & $\%$ & $\mathbf{n}$ & $\%$ & $\mathbf{n}$ & $\%$ & $\mathbf{n}$ & $\%$ & $\mathbf{n}$ & $\%$ & $\mathbf{n}$ & $\%$ & $\mathbf{n}$ & $\%$ \\
\hline $31-43$ & 14 & 27,5 & 9 & 64,3 & 2 & 14,3 & 1 & 7,1 & -- & -- & -- & -- & 2 & 14,3 \\
\hline 43-55 & 10 & 19,6 & 2 & 20,0 & -- & -- & 6 & 60,0 & -- & -- & 2 & 20,0 & -- & -- \\
\hline $56-68$ & 14 & 27,5 & 4 & 28,6 & 1 & 7,1 & 2 & 14,3 & 1 & 7,1 & 5 & 35,7 & 1 & 7,1 \\
\hline 69-85 & 13 & 25,5 & 4 & 30,8 & 2 & 15,4 & 2 & 15,4 & 1 & 7,7 & 1 & 7,7 & 3 & 23,1 \\
\hline Total & 51 & 100 & 19 & 37,3 & 5 & 9,8 & 11 & 21,6 & 2 & 3,9 & 8 & 15,7 & 6 & 11,8 \\
\hline
\end{tabular}

Teste $x^{2}(p=0,042)$

\section{DISCUSSÃO}

A análise dos resultados da avaliação sensorial mostrou que a intervenção educativa influenciou os hábitos das crianças, pois houve aumento significativo na aceitação das preparações fontes de ferro e vitamina $C$ oferecidas pelo cardápio. Estudo realizado por Lowe et al $(2004)^{20}$, que avaliou a aceitação de frutas e verduras em pré-escolares, antes, durante e após intervenção educativa, encontrou resultado semelhante apresentando maior consumo durante a intervenção do que antes da mesma.

Em relação à avaliação dos conhecimentos transmitidos, a utilização do desenho infantil mostrou que as crianças aumentaram o seu 
entendimento sobre o tema, sinalizando um comportamento positivo sobre o que fazer para ter uma alimentação mais saudável e de combate à anemia. Lima e Carvalho (2008) ${ }^{21}$ também utilizaram o desenho infantil como instrumento de avaliação da construção do conhecimento. Para os autores, normalmente os desenhos são utilizados no diagnóstico das condições psicossociais das crianças. Pode-se afirmar que os desenhos representam seus pensamentos, seus conhecimentos e/ou suas interpretações sobre uma dada situação vivida ou imaginada, com o objetivo de traduzir seu conhecimento, criando um modo simbólico de objetivação de seu pensamento.

Quando indagadas sobre como combater a anemia, a maioria (51\%) relacionou positivamente uma das abordagens utilizadas durante a intervenção para essa resposta. Vale salientar que dentre as crianças que não souberam responder à pergunta $(37,3 \%)$, a maioria apresentava idade inferior a 43 meses, podendo-se considerar as dificuldades de verbalização formal nessa idade (Tabela 4).

De acordo com Malmberga et al (2011)22, a utilização de uma metodologia de aprendizagem que respeite as peculiaridades de cada fase da vida é fundamental para se atingir os objetivos da educação nutricional. À medida que se conhece melhor os determinantes do comportamento alimentar, sejam do indivíduo ou de um grupo populacional, as chances de impacto positivo são maiores. ${ }^{14}$

No presente estudo a utilização de estratégia lúdica mostrou contribuir para a assimilação de conceitos mais abstratos como os nutrientes, suas fontes alimentares e efeitos sobre o corpo. Estudo realizado por Plum (1997) ${ }^{19}$ avaliou os conhecimentos de nutrição de pré-escolares sobre alguns alimentos, sua identificação, classificação (animal ou vegetal), origem, forma de preparo e efeito no corpo. Observou-se um percentual de $95 \%$ das crianças com três anos que identificaram corretamente os vegetais. Quanto ao efeito desses alimentos no corpo, as mesmas tiveram respostas categorizadas em ideias semelhantes às encontradas na presente pesquisa, como "bons para o corpo", "grande e forte", "sentir-se bem", "cuidar do corpo" e "saúde e crescimento".

Modelos que utilizam personagens do imaginário infantil colaboram para a percepção da criança quanto à reprodução do comportamento, principalmente quando ela tem afinidade. $\mathrm{O}$ uso de personagens de desenho animado tem se mostrado efetivo nas mudanças de hábitos alimentares ${ }^{23,24,25}$. Outra maneira de influenciar os hábitos das crianças está na utilização de reforços, ou seja, uma consequência do comportamento que aumente a probabilidade de ocorrência do mesmo comportamento ${ }^{26}$. Estudos utilizando o uso de reforçamento positivo evidenciam um aumento de consumo de frutas e verduras. ${ }^{20,23,25}$

A interação das crianças com os personagens Nutri-Heróis e o vilão Pavoroso Anêmico permitiu associação positiva com os nutrientes, e negativa em relação à doença. Observou-se identificação dos pré-escolares com seus heróis e esforço na reprodução de suas ações, como para o maior consumo de alimentos fontes de ferro e vitamina C. Bisoli e Lanzillotti (1997) ${ }^{27}$, ao avaliarem uma proposta de educação nutricional com pré-escolares do Rio de Janeiro, que teve os nutrientes também apresentados como heróis, observaram que os pré-escolares conheciam os personagens com suas respectivas imagens, citando a função de cada um, mostrando que a população alvo assimilou favoravelmente o conteúdo.

É possível perceber, desta maneira, que uma parte significativa daquilo que o sujeito aprende resulta da imitação, modelagem ou aprendizagem observacional, havendo uma complexa interação entre o ambiente, a pessoa e o comportamento. Isso permite uma grande capacidade de adaptação e aplicação ao contexto escolar através da conduta do educador e do comportamento junto aos alunos ${ }^{16,17}$.

Portanto, é fundamental a busca mais efetiva por metodologias que respeitem as características específicas de cada público alvo, considerando as etapas de desenvolvimento e maturação cognitiva. A conscientização sobre hábitos 
alimentares desde a infância é primordial na prevenção das doenças.

A eficácia de ações em educação nutricional deve estar embasada não somente na transmissão de conhecimentos específicos, mas em estratégias centradas nas necessidades motivacionais do indivíduo e sua relação social comportamento e atitude. Considera-se ainda que intervenções que abranjam uma maior parcela da população são necessárias como políticas de saúde, avaliando-se os benefícios de seus resultados.

\section{REFERÊNCIAS} edu.br:8080/revista/index.php/reci/article/view/135

\section{CONCLUSÃO}

A abordagem de estratégia nutricional para promover a alimentação saudável e prevenir a anemia em crianças, mostrou que foi efetiva em influenciar positivamente o comportamento das crianças, promovendo uma mudança no hábito alimentar para o consumo de alimentos mais variados e ricos em frutas e vegetais, bem como em alimentos fontes de ferro.

A participação e envolvimento da escola e pais das crianças nesse processo mostrou ter forte contribuição para a consolidação dessa mudança no hábito das crianças e com boa possibilidade de ser estendido ao ambiente familiar e incorporado ao hábito alimentar da família.

\footnotetext{
AGRADECIMENTOS: Agradecemos à pré-escola, aos professores e famílias (pais, cuidadores e crianças) que proporcionaram essa experiência, ao Mestrado de Nutrição e Saúde da Universidade Estadual do Ceará (UECE), que possibilitou a realização da dissertação cujos dados originaram o presente estudo, ao Laboratório de Segurança Alimentar e Nutricional e ao Conselho Nacional de Pesquisa e Desenvolvimento Científico e Tecnológico (CNPq). Em especial, a participação dos alunos do curso de Nutrição da UECE que se dedicaram com afinco à realização dessa pesquisa.
}

1. Lanes DVC, Santos MET; Silva EFSJ; Lanes KG; Folmer RLPV. Estratégias lúdicas para a construção de hábitos alimentares saudáveis na educação infantil. Revista Ciências \& Ideias, 2012; 4(1): 1-12. Disponível em: http://revistascientificas.ifrj.

2. D'Onise K, Lynch JW, Sawyer MG, McDermott RA. Can preschool improve child health outcomes? A systematic review. Social Science \& Medicine.2010; 70(9): 1423-1440. DOI:10.1016/j.socscimed.2009.12.037

3. Souza OF; Macedo LF; Oliveira CSM; Araújo TS; Muniz PT. Anaemia in children from Rio Branco, AC: prevalence and associated factors. Journal of Human Growth and Development 2012; 22(3): 307-313. DOI: 10.1590/S0102-311X2011000500018 4. Briley M, McAllaster M. Position of the American Dietetic Association: Benchmarks for nutrition programs in child care settings. J Am Diet Assoc. 2011. 111(4):607-615. DOI: 10.1016/j.jada.2011.02.016

5. Oliveira JC, Costa SD, Rocha SMB. Educação nutricional com atividade lúdica para escolares da rede municipal de ensino de Curitiba. Cadernos da Escola de Saúde [Internet], 2013. 9(1): 150-166. Disponível em: http://www.unibrasil. com.br/pdf/nutricao/2011-1/2_tcc.pdf.

6. Arcanjo FPN, Pinto VPT, Coelho MRO, Amancio MS, Magalhaes SMM. Anemia Reduction in Preschool Children with the Addition of Low Doses of Iron to School Meals. Journal of Tropical Pediatrics. 2008; 54(4): 243-247. DOI:10.1093/ tropej/fmm113

7. Kammi KS. Family structure and child anemia in Mexico. Social Science \& Medicine. 2012; 1-8. DOI: 10.1016/j. socscimed.2012.10.028.

8. Prelip M, Kinsler J, Thai CL, Erausquin JT, Slusser W. Evaluation of a School-based Multicomponent Nutrition Education Program to Improve Young Children's Fruit and Vegetable Consumption. Journal of Nutrition Education and Behavior. 2012; 44(4): 310-318. DOI:10.1016/j.jneb.2011.10.005

9. Salvi C, Ceni GC. Educação nutricional para pré escolares da associação creche Madre Alix. Revista eletrônica de extensão: Vivências. 2009; 5(8): 71-76. Disponível em: http://www.reitoria.uri.br/ vivencias/Numero_008/artigos/artigos_ vivencias_08/Artigo_33.pdf

10. Watts SO, Pinero DJ, Alter MM, Lancaster KJ. An Assessment of Nutrition Education in Selected Countiesin New York State Elementary Schools (Kindergarten through Fifth Grade). Journal of Nutrition Education and Behavior. 2012; 44(6): 474-480. DOI: 10.1016/j.jneb.2012.01.010

11. Carvalho AP, Oliveira VB, Santos LC. Hábitos alimentares e práticas de educação nutricional: atenção a crianças de uma escola municipal de Belo Horizonte, Minas Gerais. Pediatria. 2010; 32(1): 20-7. Disponível em: http://www.nutricaoemfoco. com.br/NetManager/documentos/habitos_alimentares_e_praticas_de_educacao_nutricional.pdf 
12. BRASIL. Ministério da Educação. Fundo Nacional de Desenvolvimento da Educação. Referências Nutricionais para o Programa Nacional de Alimentação Escolar. Documento Final referente ao Grupo de Trabalho revisado pelo Centro de Referência. 2009. Disponível em: http://www.crn1.org.br/images/pdf/publica/mat_tec_nut.pdf

13. Baskale H; Bahar Z. Use of Piaget's theory in preschool nutrition education. Revista Nutrição. 2009; 22 (6): $905-917$. Disponível em: http://www.scielo.br/pdf/rn/v22n6/v22n6a12.pdf

14. Souza MM. Avaliação de Programas Sociais: Um estudo sobre os efeitos de um programa de educação alimentar visando à melhoria dos hábitos alimentares. [Tese de doutorado] Brasília, Distrito Federal 2010. Disponível em: http://repositorio. unb.br/handle/10482/8327

15. Vasconcelos C; Praia JF; Almeida LS. Theory of learninf and the teaching-learning of sciences-from instruction to apprenticeship. Revista Psicologia Escolar e Educacional 2003; 7(1): 11-19. DOI: 10.1590/S1413-85572003000100002.

16. Nicolet CM, Theunissena KT. Models and theories in studies on educating and counseling children about physical health: a systematic review. Patient Education and Counseling. 2004; 55 (3): 316-330. DOI: 10.1016/j.pec.2004.08.016 17. Mohamadi FS, Asadzadeh H, Ahadi H, Jomehri F. Testing Bandura's Theory in school. Procedia Social and Behavioral Sciences. 2011; 12: 426-435. DOI: 10.1016/j.sbspro.2011.02.053

18. Jorge IMG. Preferência e grau de aceitação de alimentos por pré-ecolares com excesso de peso, matriculados em creches e pré-escolas da COSEAS/USP. [Dissertação de Mestrado]. São Paulo. 2006. Disponível em: http://www.teses.usp.br/ teses/disponiveis/6/6133/tde-08042011-094353/pt-br.php

19. Plum, JM. Nutrition knowledge assessment of Preschool children. [Tese de doutorado]. Faculty of the Virginia Polytechnic Institute and State University. Blacksburg, Virginia, 1997. Disponível em: http://scholar.lib.vt.edu/theses/available/ etd-101397-204434/unrestricted/etd.pdf

20. Lowe CF, Horne PJ, Tapper K, Bowdery M, Egerton C. Effects of a peer modeling and rewards-based intervention to increase fruit and vegetable consumption in children. Europan Journal of Clinical Nutrition. 2004; 58(3): 510-522. DOI: 10.1038/sj.ejcn.1601838

21. Lima MCB, Carvalho AMP. The infantile drawing as an evaluation instrument of the construction of physics'knowledge. Revista Electrónica de Enseñanza de las Ciencias. 2008; 7(2): 1-12. Disponível em: http://reec.uvigo.es/volumenes/volumen7/ ART4_Vol7_N2.pdf

22. Malmberga LE, Mwaurab P, Sylva K. Effects of a preschool intervention on cognitive development among East-African preschool children: A flexibly time-coded growth model. Early Childhood Research Quarterly. 2011; 26(1): 124-133. DOI:10.1016/j.ecresq.2010.04.003

23. Woolner J. Children's Food Preferences: A Behavioural Analysis. [Tese de doutorado]. University of Wales, Bangor, 2000. Disponível em: http://europepmc.org/abstract/ETH/252401

24. Bezbaruah N, Brunt A. The Influence of Cartoon Character Advertising on Fruit and Vegetable Preferences of 9-to 11-Year-Old Children. Journal of Nutrition Education and Behavior. 2012; 44(5): 438-441. DOI: 10.1016/j.jneb.2011.03.139 25. Wengreen HJ.; Madden GJ.; Aguilar SS.; Smits RR.; Jones BA. Incentivizing Children's Fruit and Vegetable Consumption: Results of a United States Pilot Study of the Food Dudes Program. Journal of Nutrition Education and Behavior. 2012;45(1): 1-6. DOI: 10.1016/j.jneb.2012.06.001

26. Abid, JA. Skinner's Moral Theory and Human Development. Psicologia: Reflexão e Crítica. 2001;14(1):107-117. DOI: 10.1590/S0102-79722001000100009

27. Bisoli MC, Lanzillotti, HS. Educação Nutricional como forma de intervenção: avaliação de uma proposta para pré-escolares. R. Nutr. Campinas.1997; 10(2): 107-113. DOI; 10.1590/S1415-52731997000200003

Recebido em 19 de janeiro de 2015. Aprovado em 06 de dezembro de 2015. 